\title{
A Method for Biometric Recognition of Ultrasound Palmprint Based on Principal Lines
}

\author{
Antonio Iula and Donatella Nardiello
}

\begin{abstract}
In this work, a method for biometric recognition of ultrasound palmprint based on principal lines is proposed and experimentally evaluated. Volumetric images of a region the palm hand were acquired by moving a commercial ultrasound probe over the region of interest. The obtained 3D matrix of voxels was then post elaborated to provide 2D renderings of the palmprint at different skin depths. The recognition procedure is based on the fusion of a couple of such images. A procedure for extracting main features from the fused image was then established and a preliminary database composed by 81 sample from 27 volunteers was created. Both verification and identification experiments were performed to evaluate system performances. Results have shown the effectiveness of the fusion procedure. Further possible improvements of the method are finally highlighted and discussed.
\end{abstract}

Index Terms-Biometrics, ultrasound, palmprint, image processing.

\section{INTRODUCTION}

Biometrics refers to methods for uniquely recognizing humans based upon one or more physical or involuntary behavioral traits [1]. Among the various biometric characteristics, which include DNA, face, hand vein, fingerprint, hand geometry, iris, retina, signature, and voice, palmprint is considered to have a good potential as a personal identification technique because it is fast and acceptable for the public. The palm of the human hand presents a lot of visual information including principal lines, wrinkles, ridges, minutiae points, singular points, texture, etc., which provide various possibilities for palmprint feature representation and pattern recognition. Palmprint research employs either high resolution or low resolution images. High resolution images (400 dpi or higher) are suitable for forensic applications such as criminal detection [2]-[4]. In this case, similar features as in fingerprints can be extracted; in addition, palmprints possess a much larger skin area and other discriminative features such as principal lines. Research on palmprint recognition mostly concentrates on low-resolution (150 dpi or lower) images, which are more suitable for civil and commercial applications such as access control. In this case, the matching is mainly based on crease and texture features [5]-[8]. Palmprint recognition techniques usually capture a two dimensional (2D) image of the palm surface and use it for

Manuscript received October 4, 2015; revised December 9, 2015. This work has been partially funded by the Italian Ministry of Education, University and Research under the project PRIN 10/11.

The authors are with School of Engineering, University of Basilicata, Potenza, Italy (e-mail: antonio.iula@unibas.it, donatella.nardiello@gmail.com). feature extraction and matching. Several techniques have been proposed for acquiring 2D palmprint images; even if 2D palmprint recognition systems generally provide excellent results in terms of accuracy and speed, they exhibit some drawbacks as 2-D palmprint images can be easily forged or affected by noise.

The most commonly used technology in biometrics is the optical one, but other techniques (capacitive, thermal and recently ultrasound) have experimented. Ultrasound has several specific advantages over other technologies. First, ultrasound systems can acquire a volumetric image of a portion of the human body, so they can provide information not only of the skin surface but also of the under skin volume. Second, Ultrasound is not sensitive to surface contaminations like stain, dirt, oil, ink and it is not affected by environmental light changes. Finally, exploiting the Doppler modality, Ultrasound can recognize life and therefore can easily detect fakes. The authors recently experimented 3D ultrasound imaging of the human hand for biometric recognition purposes. Several biometric characteristics have been extracted from the analysis of a 3D volumetric ultrasound image, including inner hand geometry [9], fingerprint [10], [11], vein pattern [12] and palmprint [13]-[16].

In this work, an ultrasound palmprint recognition system is proposed and evaluated. The method is based on the acquisition of a couple of $2 \mathrm{D}$ palmprints at different under skin depth. The proposed method is evaluated by performing verification and identification experiments by exploiting a database of 3D ultrasound images.

The remainder of the paper is organized as follows: Section II describes the ultrasound technique exploited for achieving 3D ultrasound images of a region of the human palm to be used for palmprint recognition. Section III describes the steps of the recognition procedure and Section IV the experimental recognition results. Conclusions are reported in Section V.

\section{3D ULTRASOUND IMAGING}

Ultrasound imaging is nowadays widely employed in several biomedical applications. Basically, ultrasound images are made by sending an ultrasound pulse into tissue by using an ultrasound transducer (probe). The sound reflects (echoes) from parts of the tissue; these echoes are recorded and displayed as an image.

Several modes of ultrasound are used in medical imaging. A-mode (amplitude mode) is the simplest type: a single transducer scans a line through the body with the echoes plotted on screen as a function of depth. In B-mode (brightness mode) ultrasound, a linear array of transducers simultaneously scans a plane through the body that can be viewed as a two-dimensional image on screen [17]. 


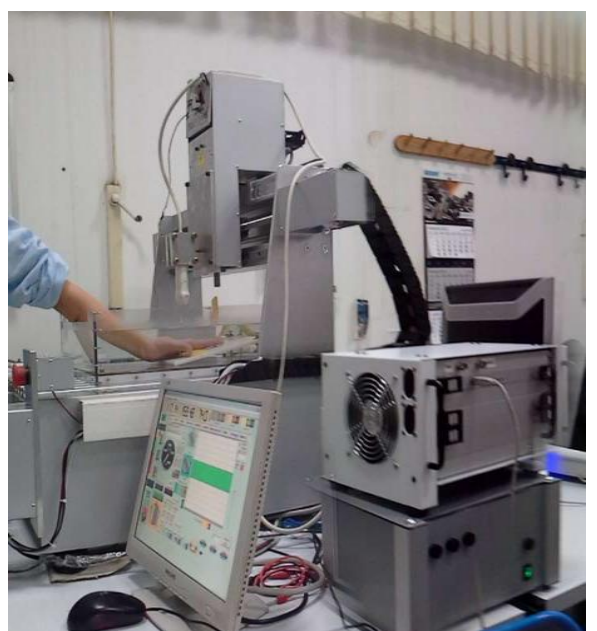

Fig. 1. A photo of the experimental set up used to acquire 3D images.

A-mode imaging has been exploited in the past for biometric purposes mainly for fingerprint recognition [18]-[20]. Basically, a 3D ultrasonic image of the under skin volume is achieved by mechanically moving a high frequency A-mode ultrasonic transducer along two directions Despite of the high achievable resolution, an important drawback in this technique relies in the very high scanning time, which would be prohibitive for applications where the area to be scanned is large.

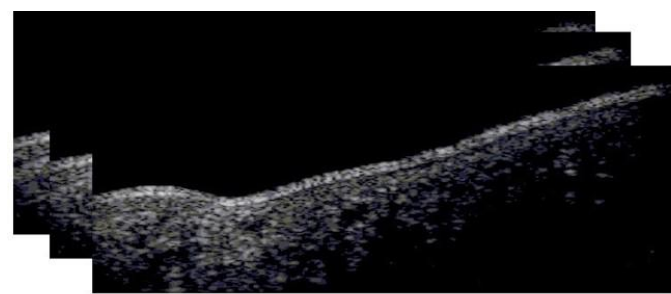

Fig. 2. Volumetric image reconstruction through the acquired B-scans.

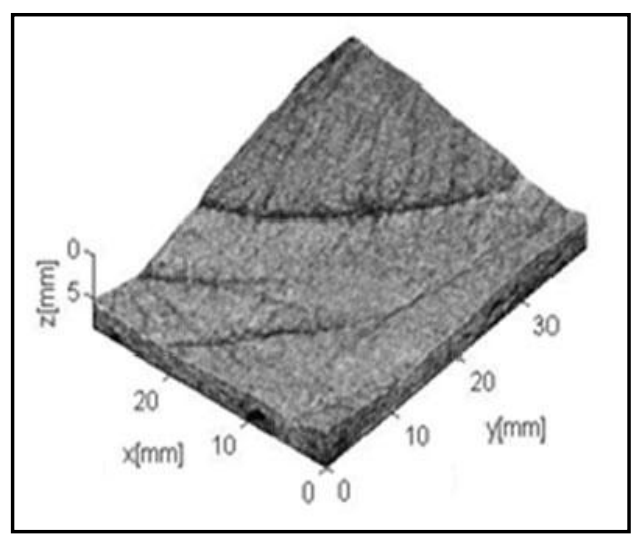

Fig. 3. 3D rendering of a region of the hand of a volunteer. Palm curvature and main traits can be appreciated.

Some of the authors recently experimented with a different ultrasound technique, based on the investigation of the human hand, which could be exploited for biometric recognition purposes: a 3D image representing a portion of the hand volume, is acquired by mechanically shifting a linear array over the region of interest [9]-[17]. The use of the array on one hand reduces the acquisition time as only one linear scan is required, on the other hand allows the use of appropriate beamforming strategies to optimize the ultrasonic images [21]. These features compensate for the smaller resolution achievable with respect to the techniques based on A-scan transducers, due to the lower maximum working frequency of the array.

The experimental setup for getting the 3D ultrasound palmprint images is shown in Fig. 1. The user places the palm of his hand on a plastic jig, which has guidance marks on it. The hand is properly aligned by the marks and is completely immersed in water with the palm facing upwards. A commercial 192 elements ultrasound array (LA435 by Esaote S.p.a., Genova, Italy) is used as ultrasound transducer; it is based on piezo composite technology and has a central working frequency of $12 \mathrm{MHz}$. The ultrasound probe is tied on a numeric controlled pantograph by Delta Macchine CNC, Vazia (RI) - ITALY, which is able to perform 3D path with a precision better than $20 \mu \mathrm{m}$. The ultrasound probe-head is partially immersed in order to be faced and acoustically coupled to the hand palm. An advanced open platform for ultrasound research (ULA OP) [22] is employed as ultrasound imaging system. The system is extremely compact as all electronics are integrated in two boards, which are contained in a box and connected to a PC through USB 2.0. The system is characterized by the full programmability of each critical section. Both the pantograph and ULA OP are controlled in MATLAB environment (The MathWorks, Inc., MA, USA).

In order to acquire 3D ultrasound imaging data of a volume of the human hand, the probe is mechanically shifted along the elevation direction while a number (typically 250) of B-mode images (B-scans) are one by one acquired and stored in an asynchronous way with respect to the continuous motion of the pantograph. With this technique, a 3D ultrasound image corresponding to a volume of $30 \times 50 \times 15$ $\mathrm{mm}^{3}$ is acquired in about 5 seconds. The volumetric image is then reconstructed in post process by simply grouping the acquired B-scans in a 3D matrix as shown in Fig. 2.

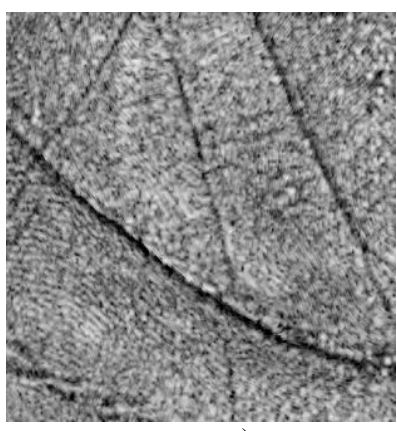

a)

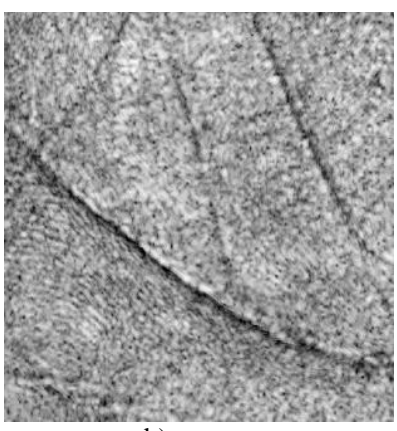

b)
Fig. 4. 2D palmprints at different under skin depths: a) $0.03 \mathrm{~mm}$, b) $0.09 \mathrm{~mm}$

An ad hoc software written in Matlab code provides several renderings that allow to appreciate 3D information of the palmprint. As an example, Fig. 3 shows a 3D rendering of a portion of a human hand where the curvature of the palm together with the principal traits can be clearly appreciated.

$2 \mathrm{D}$ renderings, i.e., projections of the palmprint on a plane (2D palmprint) can be also achieved. Fig. 4 shows two of such images at two different under skin depths. This is an important peculiarity of the ultrasound, which cannot be found in classical 2D optical palmprints.

\section{RECOGNITION PROCEDURE}

The proposed recognition method is based on the extraction of the main features from 2D ultrasound images of 
the palm. These are grey scale images with a dimension of $512 \times 676$ pixels.

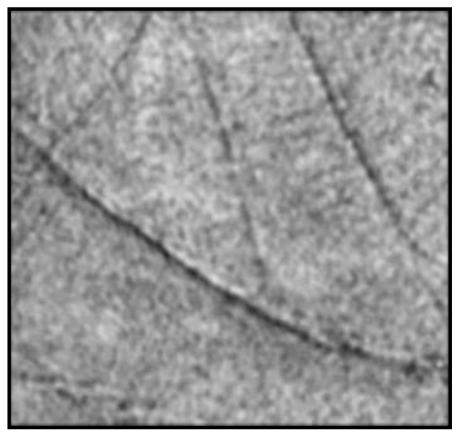

Fig. 5. Resulting image from the fusion of the images of Fig. 4.

\section{A. Images Fusion}

The procedure takes as input the two 2D images shown in Fig. 4, which are at different under skin depth, i.e. $0.03 \mathrm{~mm}$ and $0.09 \mathrm{~mm}$. Each images is smoothed by applying an opportune filter. Successively, the two images are fused by applying a 3D filter, which, basically, correct pixel regions of the deeper image that are darken than the corresponding ones in the other image. This correction is applied because, in ultrasound images, principal lines become brighter and brighter as they go through the skin. Overall, 3D filtering operate a mean between the two source images. The resulting image is shown in Fig. 5.

\section{B. Features Extraction}

In order to extract main features, i.e., those corresponding to principal lines, an hoc procedure was developed.
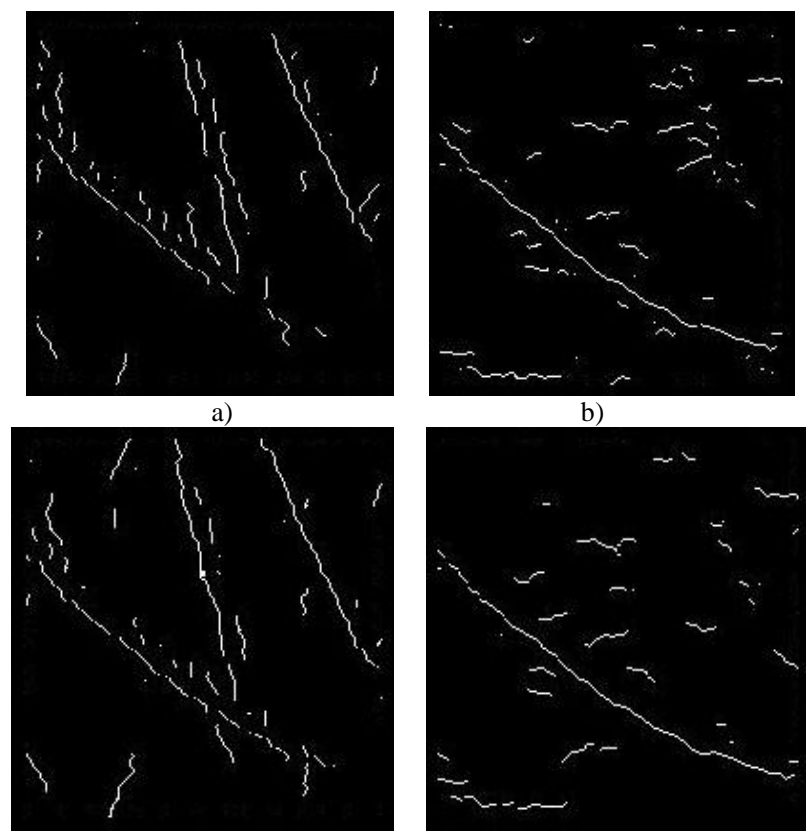

c)

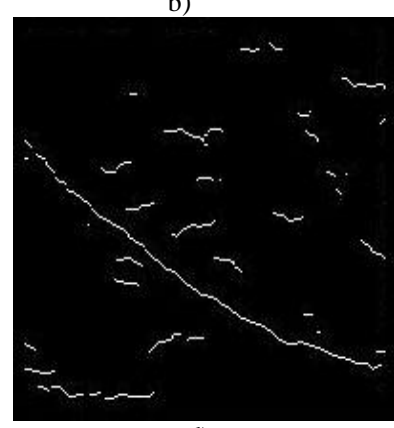

d)

Fig. 6. Features extraction by scanning the input image a) from left-to right, b) from the bottom up, c) from right to left, d) from top to bottom.

The procedure is able to detect all points of the matrix that correspond to significant variation of grey levels. The image is scanned along four directions, i.e., from left to right, from the bottom up, from right to left and from top to bottom. Each scan is able to detect mainly palm lines that are more or less orthogonal to the scan direction.

Fig. 6 shows the four resulting images after binarization.
The four images are then combined through a logical sum operation to provide a final image (see Fig. 7) that contains all extracted features.

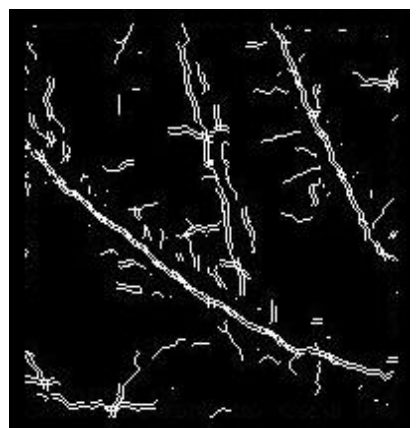

Fig. 7. Image obtained with a logical sum of the images of Fig. 6.

\section{Template Extraction}

In order to obtain the final template that is used in the matching phase, some morphological operations like closing and thinning were performed. Further elaborations aimed to eliminate short traits were finally executed. Fig. 8 shows the resulting template a) alone and b) superimposed to the source palm image. As can be seen the extracted template reproduces quite accurately the principal lines of the palm.
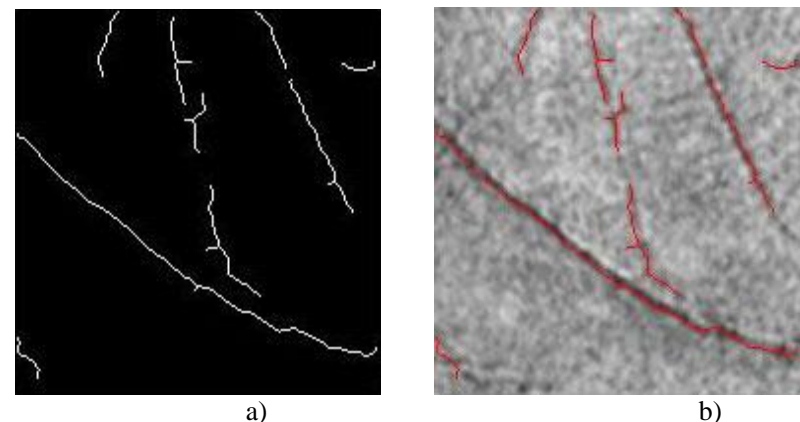

b)

Fig. 8. Template a) alone and b) superimposed to the palm image.

\section{EXPERIMENTAL RESULTS}

With the aim of providing an evaluation of the proposed 3D palmprint recognition system, an experimental database was established. The database contains from 81 samples from 27 users, i.e., three samples were collected from each user at different times.

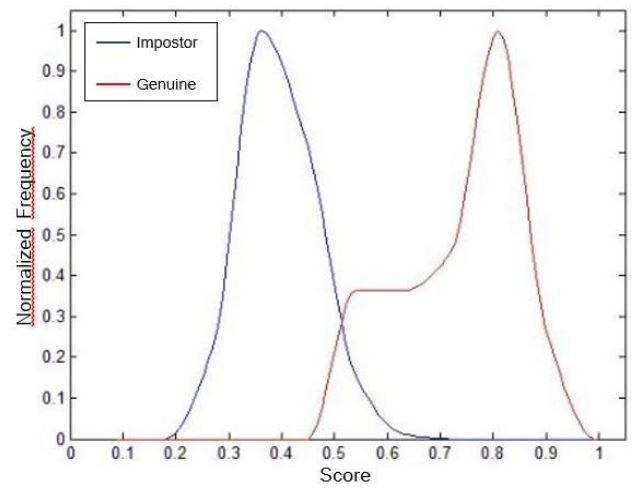

Fig. 9. Genuine and impostor distributions for the fused image.

The method used to implement the matching is the so called pixel-to-area matching [5]. A matching score quantitatively expresses the similarity between the features of two binary images. To calculate this value, the following 
mathematical expression was used:

$$
S(A, B)=\frac{2}{M_{A}+M_{B}} \times \sum_{i=1}^{M} \sum_{j=1}^{N}\left[A_{D}(i, j) \cap B(i, j)\right]
$$

where $A$ and $B$ are the binarized images, $A_{D}$ is the matrix $A$ dilated, $M \times N$ is the size of the matrix $A$, " $\cap$ " is the operation of logical AND, $M_{A}$ and $M_{B}$ are numbers of non-zero pixels in $\mathrm{A}$ and $\mathrm{B}$.

In order to account for a small mispositioning of the user's palm during successive acquisitions, translations and rotations are performed on the image and a matching score is computed for each operation; therefore, the maximum score is the selected one.

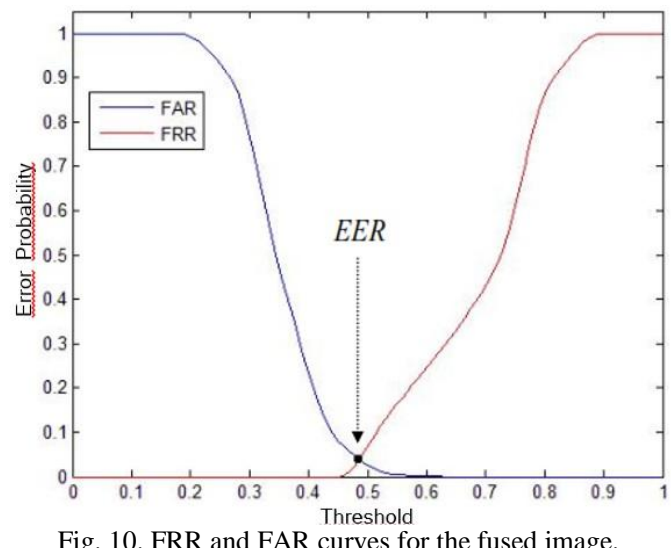

Genuine scores are scores that represent comparisons of different images that belong to the same person, instead, impostor scores are scores that represent comparisons of images that belong to different persons. Fig. 9 shows the normalized genuine and impostor distributions for the fused image. The overlapped region of genuine and impostor distributions is the source of two type of errors: FAR (False Acceptance Rate) and FRR (False Rejection Rate). These two errors belong to all real biometric systems. Fig. 10 shows the FAR and FRR curves as a function of the threshold for the fused image. The decision threshold of the system depends on which of the two errors one wants to minimize. A parameter often used to compare different algorithms is EER (Equal Error Rate), i.e., the error obtained if the threshold is set to have FAR $=$ FRR.

TABLE I: EER AND THRESHOLD VALUES

\begin{tabular}{|l|l|l|}
\hline & Threshold & EER \\
\hline Image at $0.03 \mathrm{~mm}$ & 0.47 & $5.7 \%$ \\
\hline Image at $0.09 \mathrm{~mm}$ & 0.47 & $4.7 \%$ \\
\hline Fused image & 0.49 & $3.7 \%$ \\
\hline
\end{tabular}

Table I reports values of the EER and the corresponding threshold. For comparison, the same parameters obtained by applying the features extraction procedure to the two single images are reported. As can be seen the fusion procedure is effective and improves the overall performance of the system.

\section{CONCLUSIONS}

In this work, an ultrasound palmprint recognition system is proposed and experimentally evaluated. A 3D image of a region of the human palm is achieved by using an ultrasound linear probe. The probe is moved along its elevation direction and successively acquires a number of 2D images (BScans), which are then grouped in a 3D matrix. The data are then processed to extract palmprint images a different under skin depths.

A procedure to extract main features of the palmprint, i.e., principal lines, was established. It allows to use as input two fused palmprint images at different depths.

In order to validate the procedure, a 3D palmprint ultrasound database with 81 samples from 27 volunteers, i.e., three samples from each subject at different times, was created. Experimental results have shown that the proposed system, even if tested on a relatively small database, exhibits acceptable recognition rates. Also, it was observed that the fusion of two images at different depths allows to decrease the recognition error with respect to the two single images.

Next work will be devoted to increase the number of fused images at different depths to improve the performance of the system and to establish a procedure that uses a $3 \mathrm{D}$ template which is able to take into account the depth of the principal lines.

\section{ACKNOWLEDGMENT}

The authors wish to thank Francesco Labriola for his helpful suggestions.

\section{REFERENCES}

[1] A. K. Jain, A. Ross, and S. Prabhakar, "An introduction to biometric recognition," IEEE Transactions on Circuits and Systems for Video Technology, vol. 14, pp. 4-20, 2004.

[2] J. Dai and J. Zhou, "Multifeature-based high-resolution palmprint recognition," IEEE Transactions on Pattern Analysis and Machine Intelligence, vol. 33, no. 5, pp. 945-957, 2011.

[3] R. Cappelli, M. Ferrara, D. Maio, "A fast and accurate palmprint recognition system based on minutiae," IEEE Trans. Syst., Man, Cybern., B, Cybern, vol. 42, no. 3, p. 956962, 2012.

[4] R. Wang et al., "Regional fusion for high-resolution palmprint recognition using spectral minutiae representation," IET Biometrics, vol. 3, no. 2, pp. 94-100, 2014.

[5] D. Zhang, Palmprint Authentication, Kluwer Academic Publishers, 2004.

[6] D. Huang, W. Jia, and D. Zhang, "Palmprint verification based on principal lines," Pattern Recognition, vol. 41, no. 4, pp. 1316-1328, 2008.

[7] A. Kong, D. Zhang, and M. Kamel, "A survey of palmprint recognition," J. Pattern Recognit., vol. 42, no. 7, pp. 1408-1418, 2009.

[8] D. Zhang, W. Zuo, and F. Yue, "A comparative study of palmprint recognition algorithms," ACM Comput. Surveys, vol. 44, no. 1, p. 2 , 2012.

[9] A. Iula and M. Santis, "Experimental evaluation of an ultrasound technique for the biometric recognition of human hand anatomic elements," Ultrasonics, vol. 51, no. 6, pp. 683-688, 2011.

[10] N. Lamberti, G. Caliano, A. Iula, and A. S. Savoia, "A high frequency cMUT probe for ultrasound imaging of fingerprints," Sensor \& Actuators A, Physical, vol. 172, pp. 561-569, 2011.

[11] A. Iula, A. Savoia, and G. Caliano, "Capacitive microfabricated ultrasonic transducers for biometric applications," Microelectronic Engineering Journal, vol. 88, pp. 2278-2280, 2011.

[12] A. Iula, A. Savoia, and G. Caliano, "3D ultrasound palm vein pattern for biometric recognition,” IEEE Int. Ultrasonics Symp., no. 6562283 , pp. 2442-2445, 2012.

[13] A. Iula, A. Savoia, and G. Caliano, "An ultrasound technique for 3D palmprint extraction," Sensor \& Actuators A, Physical, vol. 212, pp. 1824,2014

[14] A. Iula, G. Hine, A. Ramalli, F. Guidi, E. Boni, A. S. Savoia, and G. Caliano, "An enhanced Ultrasound technique for 3D Palmprint Recognition,” IEEE Int. Ultrasonics Symp., pp. 978-981, 2013. 
[15] A. Iula, G. Hine, A. Ramalli, and F. Guidi, "Wide 3D ultrasound palmprint for biometric recognition," in Proc. IEEE International Ultrasonics Symposium, IUS, no. 6931989, pp. 1388-1391, 2014.

[16] A. Iula, G. Hine, A. Ramalli, and F. Guidi, "An improved ultrasound system for biometric recognition based on hand geometry and palmprint," Procedia Engineering, 87, pp. 1338-1341, 2014.

[17] H. Azhari, Basics of Biomedical Ultrasound for Engineers, John Wiley \& Sons, 2010.

[18] J. K. Schneider and S. M. Gojevic, "Ultrasonic imaging systems for personal identification," in Proc. IEEE Int. Ultrasonics Symp., pp 595-601, 2001

[19] R. M. Schmitt, J. Zeichman, A. C. Casanova, and D. Delong, "Model based development of a commercial, acoustic fingerprint sensor," in Proc. IEEE Int. Ultrasonics Symp., no. 6562410, pp. 1075-1085, 2012.

[20] R.G Maev and F. Severin, "High-speed biometrics ultrasonic system for 3D fingerprint imaging," in Proc. SPIE - The International Society for Optical Engineering, vol. 8546, no. 85460B, 2012.

[21] A. Fenster, D. B. Downey, and H. N. Cardinal, "Three-dimensional ultrasound imaging," Physics in Medicine and Biology, vol. 46, pp. 67-99, 2001.

[22] P. Tortoli et al., "An advanced open platform for ultrasound research," IEEE Trans. Ultrason., Ferroelectr., Freq. Control, vol. 56, no. 10, p. 22072216, 2009

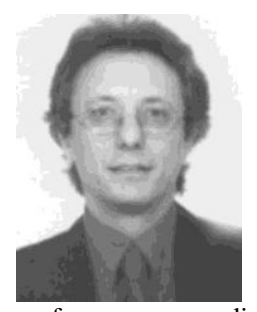

Antonio Iula is an associate professor of electronics at the University of Basilicata, Potenza, Italy. His research activity is mainly devoted to devices and systems for the application of ultrasounds in both biomedical (diagnostic imaging, surgery, therapy, biometry) and industrial fields (non destructive testing, ultrasonic motors and actuators, sensors). He is the author of more than 90 papers in these fields that have been published in international journals and conferences proceedings, and he has been involved in several national and international research projects.

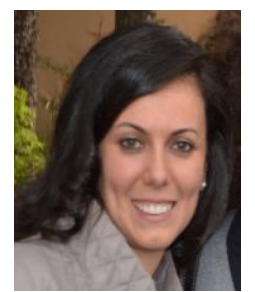

Donatella Nardiello received the MS degree in computer and information engineering in 2014 at the University of Basilicata, Potenza, Italy. Her main research interests are ultrasound imaging and image processing. 